\title{
Heavy-ion physics
}

\section{Giant nucleus at Darmstadt?}

\section{from Joshua Silver}

WHEN an energetic very heavy ion passes close to the nucleus of a heavy atom in a solid target, positrons may be spontaneously produced within the electron shells of the atom. Recently, researchers at the heavy-ion facility at GSI Darmstadt, West Germany, have been examining this phenomenon. One particularly exciting possibility emerging from these experiments is that extremely heavy nuclei, with atomic number and mass of about 180 and 470 respectively, might be produced in heavy-ion scatterings. Although such products would be short lived, they might open up the study of atoms with unprecedented properties.

Consider an atom of variable nuclear charge $Z$. As $Z$ increases, the binding energy of the innermost electrons, $E(1 \mathrm{~s})$, becomes more negative. Forgetting for a moment the fact that there are no atoms with $Z>109$, we may calculate $E(1 \mathrm{~s})$ using the Dirac equation with a Coulomb potential for arbitrary $Z$. It is found ${ }^{1-3}$ that for a non-

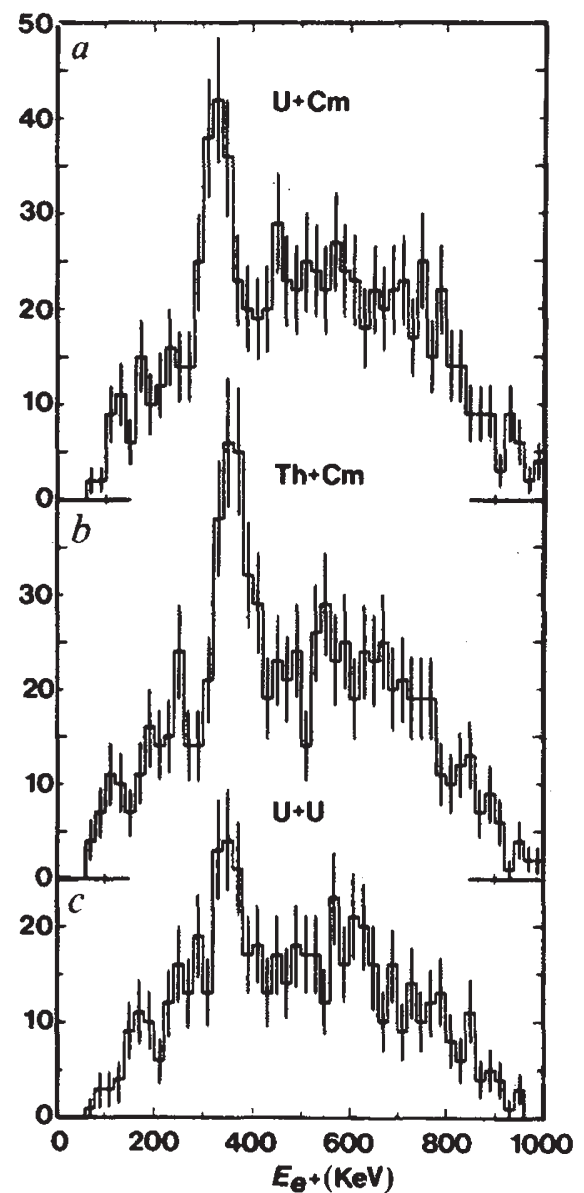

Fig. 1 Energy spectra of positrons produced from collisions of $a,{ }^{23 x} \mathrm{U}(Z=92)$ on ${ }^{23 x} \mathrm{Cm}(Z=96)$; b, ${ }^{232} \mathrm{Th}(Z=90)$ on ${ }^{2+8} \mathrm{CM}(Z=96) ; c,{ }^{238} \mathrm{U}(Z$ =92). Bombarding energies are $\sim 6 \mathrm{MeV} \mathrm{A}$ and the angular range (of the order of 100) of the ions scattered in coincidence with the detected positrons gives the most pronounced $\sim 300 \mathrm{keV}$ positron 'line' in each case (from ref. 7). zero nuclear charge radius, $E(1 \mathrm{~s})$ falls below $-2 m_{e} c^{2}$ for $Z \geqslant 173$, where $m_{e}$ is the rest mass of the electron and $c$ is the velocity of light. When $E(1 \mathrm{~s})$ is less than $-2 m_{e} c^{2}$, the discrete bound electron state becomes degenerate in energy with a three-particle continuum state, consisting of two bound electrons plus an outgoing positron wave.

It was first suggested by Gershtein and Zel'dovich' that this 'autoionizing' mode of spontaneous positron production might be observable in heavy-ion collisions. The idea is that for a sufficiently close collision of two very heavy ions, it is possible for the 'innermost' is electrons of the projectile or target atom to experience a Coulomb field similar to that from a nucleus with $Z \geqslant 173$, so long as $Z_{1}+Z_{2} \geqslant 173$, where $Z_{1}$ is the atomic number of the projectile ion and $Z_{2}$ the atomic number of the target atom ${ }^{4}$. This approach, however, suffers from a weakness - in the words of Müller ${ }^{5}$, the method is "both a curse and a gift"; the rapid time variation of the Coulomb field during the collision leads to ionization of $1 \mathrm{~s}$ electrons from the collision partners, thereby creating vacancies. This is the "gift", in that spontaneous production of positrons is suppressed by the Pauli exclusion principle if the 1s levels are full. However, the time variation of the Coulomb field is also responsible for induced positron production which can mask the spontaneous process. Calculation of the relative probabilities of the spontaneous and induced processes is difficult; for completely elastic scattering along Rutherford trajectories the two processes cannot be distinguished in principle. There is also a contribution to the total positron production from nuclear mechanisms.

Three experimental groups have used the heavy-ion accelerator at GSI Darmstadt for several years to search for spontaneously produced positrons. In the past two years, these groups have observed a new phenomenon that is difficult to explain ${ }^{6 \cdot 8}$. For coincident observation of positrons with particles scattered in well-defined angular ranges, and at well-defined ion-beam energies in a narrow interval around the Coulomb barrier, the energy spectra of the positrons produced during a collision can show a distinct 'line' at around 300 keV.Typical spectra are shown in Fig. 1. The width of this line may be used to infer a duration for the collision, simply from Heisenberg's energy-time uncertainty relation. The smallest widths observed so far (about $60 \mathrm{keV}$ ) translate into a characteristic time for the positron production process of the order of $10^{-19}$ seconds. Now it is also possible, from the dynamics of the collision, to estimate the time during which the collision partners are sufficiently close together for the transient 'united atom' Coulomb ()1985 Nature Publishing Group

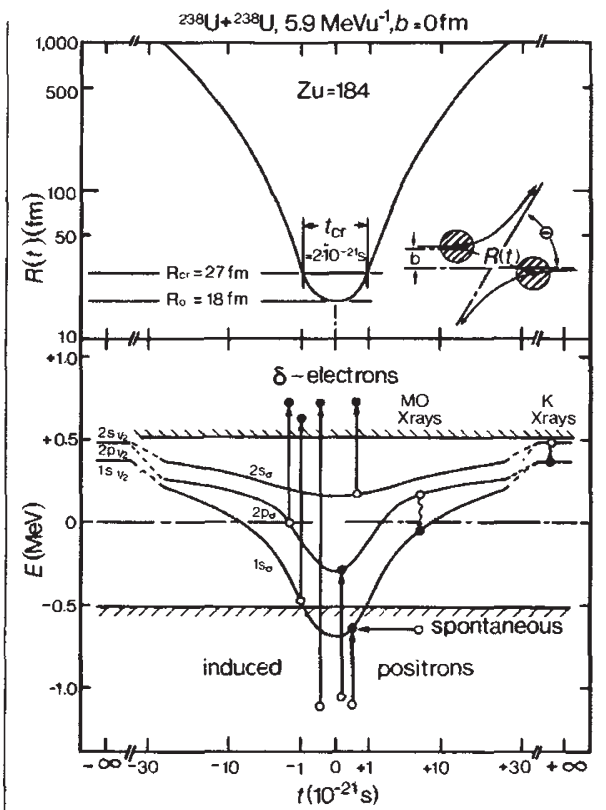

Fig. 2. Electron-binding energies, $t$, for the three most bound states (top) and the internuclear separation, $R(t)$, for two ${ }^{23 k} \mathrm{U}$ nuclei as a function. of time (bottom) for a collision between a stationary target nucleus and a projectile with energy $1 \cdot 40 \mathrm{GeV}$, with impact parameter $b=0$. Note that $E(1 \mathrm{~s})$ is $<-2 m_{\mathrm{e}} \mathrm{c}^{2}$ for a time of the order of $2 \times 10^{21} \mathrm{~s}$ for this collision (from ref. 10).

field to be large enough to give rise to spontaneous positron emission. For the typical system ${ }^{238} U+{ }^{238} U$ at a beam energy of $1.40 \mathrm{GeV}$ and a head-on collision, this time is $-10^{-21}$ seconds, two orders less than the duration inferred from the width of the positron line (Fig. 2). What is going on?

One possible explanation is the formation, during the collision, of a transient 'giant di-nuclear system' or 'giant nuclear molecule' which lasts for at least $10^{-19}$ seconds. At present, it is not clear that this explanation is correct or complete, and one problem that is not easily explained is that the most well-defined positron lines are all at an energy of about $300 \mathrm{keV}$. Nonetheless, the possible creation of an (albeit transient) nuclear system with an atomic mass of about 470 and an atomic number of around 180 is most exciting and has triggered more experimental and theoretical work, in an attempt to understand what is really happening. With the increasing availability of heavyion beams of suitable energy it is likely that this phenomenon will soon be better understood.

\footnotetext{
1. Gershtein, S.S. \& Zel'dorich, Ya.B. Nuovo Cimento Lent $835(1969)$.

3. Zel'dovich, Ya.B. \& Popos, Y.S. Lipp. M... Nank 105, 373 (1972).

. Nuller, B. Rafelski. J. \& Greiner. W. 2. Phys. 275, 62, 18 (1972)

4. Brodsky, S. \& Mlohr, P. in Structure and (ollisioms of loms and Atoms (ed. Sellin, I.A.) (Springer, Berlin, 197X)

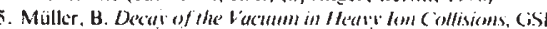
preprint 84-62 (1984); Pros. Tha'rer. Phys. (in the pros)

6. Schweppe, J. of al. Phys. Res. teth. 51. 2261 (1983).

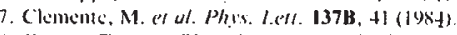

8. Cowan, T. e' al. Phys. Re's ledf. (submitted).

9. Reinhardı, J. of al. \%. Ph/s A31)3, 173 (1981).

10. Bokemeyer, H. Positron Sipectroscony of Supercritical

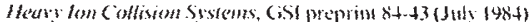

Joshua Silver is at the Clarendon Laboratory, University of Oxford, Parks Road, Oxford $O X 13 P U, U K$. 